\title{
A VANISHING THEOREM FOR THE CONFORMAL ANOMALY IN DIMENSION $>2$
}

\author{
JACK MORAVA AND HIROTAKA TAMANOI
}

\begin{abstract}
We examine the conformal anomaly of quantum field theory in the light of Atiyah and Singer's recent work on the chiral anomaly. We show that the bundle over the space of conformal structures defined on a given manifold, constructed as the index of the conformally invariant family of Laplace operators, is a torsion class in real $K$-theory, Indeed, its Chern forms are zero.

These Chern forms transgress to define classes in certain local cohomology groups calculated recently by Bonora, Cotta-Ramusino, and Reina; a corollary is that, although these obstruction groups are nonzero, the obstruction to the existence of a gauge-covariant propagator for the Laplace operator is the trivial element of obstruction group.
\end{abstract}

\section{Introduction.}

1.0. In what follows $M$ is a compact orientable smooth manifold of dimension $n>2$, and $\mathfrak{M}$ will denote the space of its Riemannian metrics. The group $\mathfrak{D}$ of diffeomorphisms of $M$ acts naturally on $\mathfrak{M}$, and if $x_{0} \in M$ is a basepoint, we write $\mathfrak{D}_{0}$ for the subgroup of diffeomorphisms leaving fixed a chosen linear basis for the tangent bundle over $x_{0}$, following [4]. The topological category (or transformation groupoid) defined by the action of $\mathfrak{D}$ on $\mathfrak{M}$ is the topic of [12].

1.1. The Weyl gauge group $\mathfrak{W}$ of smooth maps from $M$ to $\mathbb{R}_{+}^{\times}$inherits a $\mathfrak{D}$-action, and the semidirect product $\mathfrak{W} \cdot \mathfrak{D}$ acts on $\mathfrak{M}$ to define the category of Riemannian metrics and conformal equivalences (cf. [15]). We follow the convention above and write $\mathfrak{W}_{0}$ for the subgroup of functions in $\mathfrak{W}$ equal to 1 at the basepoint; we denote by $\mathfrak{C}$ the quotient $\mathfrak{W} \backslash \mathfrak{M}$ and by $\mathfrak{C}_{0}$ the quotient $\mathfrak{W}_{0} \backslash \mathfrak{M}$. Then $\mathfrak{C}$ is a $\mathfrak{D}$-space, and $\mathfrak{C}_{0}$ is a $\mathfrak{D}_{0}$-space with free action.

1.2. Here we will be concerned with the $\mathfrak{D}$-equivariant family

$$
Y(g)=*_{g}^{2 / n}\left(-\Delta_{g}+\frac{1}{4} \frac{n-2}{n-1} R(g)\right)
$$

of elliptic operators parametrized by $\mathfrak{C}$ constructed in 1960 by $\mathrm{H}$. Yamabe. There are some technical details needed for its construction, which can deferred until $\S 2$.

Our work is modelled on the study of Dirac operators parametrized by the potentials of a gauge field; in that case Atiyah and Singer exhibit a $K$-theoretic obstruction ind $\not \partial$ to the existence of a gauge-covariant inverse, or Feynman propagator, for the Dirac operator, and calculate the transgressed first Chern class (see $\S 1.4$ ) $T c_{1}$ (ind $\not$ ) in terms of a cocycle that had earlier been constructed by Bardeen, as a kind of radiative correction to the stress tensor (cf. [7]). Our principal conclusion here is that although the conformal anomaly is similar in many ways to the chiral

Received by the editors November 20, 1985 and, in revised form, April 18, 1986.

1980 Mathematics Subject Classification (1985 Revision). Primary 55R50, 57M35, 57R50, 58D30, $58 \mathrm{G} 10$. 
anomaly (cf. [16]), in other ways it is simpler. In particular all obstructions to the existence of a covariant Feynman propagator for $Y(g)$ are torsion (cf. [17, 22]).

1.3. Now $\S 1.1$ can be paraphrased by saying that $\mathfrak{M}$ is a $\mathfrak{D}$ - (resp. $\mathfrak{D}_{0^{-}}$) equivariant principal bundle over $\mathfrak{C}$ (resp. $\left.\mathfrak{C}_{0}\right)$, with structure group $\mathfrak{W}$ (resp. $\left.\mathfrak{W}_{0}\right)$. This is a little unlike the gauge groups of Yang-Mills theory, in that $\mathbb{R}_{+}^{\times}$is (i) noncompact and (ii) contractible. Nevertheless, it has nontrivial Lie algebra cohomology.

If, in general, $\pi: P \rightarrow B$ is a principal bundle with structure group $G$, then a closed differential form $c$ on $B$ which pulls back to an exact form $\pi^{*} c=d T c$, e.g. a characteristic class of $P$ (cf. [11]), defines an invariant

$$
[T c] \in H^{k}\left(\mathfrak{g}, \Omega^{0} P\right)
$$

in the cohomology of the Lie algebra $\mathfrak{g}$ of $G$, according to the formula

$$
\Omega^{k} \mathfrak{g} \rightarrow \Omega^{0} P: v_{1}, \ldots, v_{k} \rightarrow(T c)\left(i_{v_{1}}, \ldots, i_{v_{k}}\right),
$$

$i_{v}$ being the lift of a left-invariant vectorfield on $G$ to a vertical vectorfield on $P$ (cf. $[14])$.

1.4. For example, the chiral anomaly ind $\not \partial$ is a virtual vector bundle on quotient $\mathfrak{A} / \mathfrak{G}$ of a space of connections by the gauge group, which pulls back to a trivial bundle on the space $\mathfrak{A}$.

Similarly, $Y(g)$ pulls back from $\mathfrak{C}$ to define a family of operators on the spaces $L^{2}\left(M, d \operatorname{vol}_{g}\right)$ parametrized by $\mathfrak{M}$. At each $g$ in $\mathfrak{M}$ the associated operator is essentially selfadjoint and bounded below, but the space of such operators is contractible [4] and ind $Y$ pulls back to a trivial bundle on $\mathfrak{M}$, which suggests $H^{\text {odd }}\left(\mathfrak{w}, \Omega^{0} \mathfrak{M}\right)$ valued invariants.

Remarkable enough, such cohomology groups have been studied recently by mathematical physicists $[\mathbf{8}, \mathbf{9}]$ interested in the conformal anomaly. In the next sectin we discuss their results in slightly greater detail.

\section{Digressions.}

2.0. The cohomology of Lie algebras defined by various large groups, e.g. of diffeomorphisms or gauge transformations, with coefficients in the algebra of forms in some infinite-dimensional manifold, e.g. $\mathfrak{M}$, upon which the group acts is called local when the sort of forms are required to be suitable locally defined. We write $\Omega_{\text {loc }}^{*}$ for the subcomplex of such forms. The results of [9] can be summarized as follows:

2.1. When $n$ is four, the $\mathfrak{D}$-equivariant elements of $H^{1}\left(\mathfrak{w}, \Omega_{\mathrm{loc}}^{0} \mathfrak{M}\right)$ form a twodimensional vectorspace, with cocycles

$$
w \rightarrow \int w c_{J}(g), \quad J=\mathrm{I}, \mathrm{II},
$$

as a basis; here

$$
c_{\mathrm{I}}=C_{i j k l} C^{i j k l} d \operatorname{vol}_{g}
$$

is the square of the Weyl tensor, and $c_{\mathrm{II}}$ is the Euler form.

2.2. There is another result of this nature which takes more notation to state, but the work is needed anyway.

We define the line bundle of real $d$-densities, $d \in \mathbb{R}$, in terms of the principal $\mathrm{Gl}_{n}$-bundle $\mathrm{Fr}$ of vector space bases for the tangent space of $M$ by the diagram

$$
\mathbb{R}(d) \times{ }_{\mathrm{Gl}} \mathrm{Fr} \rightarrow \mathrm{pt} \times \times_{\mathrm{Gl}} \mathrm{Fr}=M,
$$


with $\mathbb{R}(d)$ the representation of $\mathrm{Gl}_{n} \mathbb{R}$ defined for $A \in \mathrm{Gl}_{n} \mathbb{R}$ by

$$
A \rightarrow \begin{cases}\operatorname{sgn} \operatorname{det} A \cdot|\operatorname{det} A|^{d / n}, & d \notin 2 Z, \\ |\operatorname{det} A|^{d / n}, & \text { otherwise. }\end{cases}
$$

The bundle of $n$-densities can then be naturally indentified with the bundle of volume-forms; and if $\sigma$ is a $d$-density, then $|\sigma|^{\otimes n / d}$ is an $n$-density. The space of such $d$-densities acquires the natural Banach-space norm

$$
\sigma \rightarrow\left[\int|\sigma|^{\otimes n / d}\right]^{d / n}=\llbracket \sigma \rrbracket .
$$

A choice $g \in \mathfrak{M}$ of Riemannian metric defines the isometry

$$
\phi \rightarrow *_{g}^{-d / n} \phi=f
$$

of the space of sections, normed by $\llbracket-\rrbracket$, to the more usual Lebesgue space $L^{n / d}\left(M, d \operatorname{vol}_{g}\right)$. (The notation is motivated by the identity

$$
\left.*_{g} 1=|\operatorname{det} g|^{1 / 2} d x_{1} \wedge \cdots \wedge d x_{n} .\right)
$$

2.3. We use $\mathbf{L}(d)$ to denote the Banach of $d$-densities. In particular, $\mathbb{L}\left(\frac{1}{2} n\right)$ is more usually called the space of $\frac{1}{2}$-densities. As justification for the funny typeface we plead that Hölder's inequality (i.e. that $\mathbb{L}\left(d_{0}\right) \otimes\left(d_{1}\right) \rightarrow \mathbb{L}\left(d_{0}+d_{1}\right)$ is continuous) can be relegated to parentheses.

Now the spaces $L\left(\frac{1}{2} n-1\right)$ and $L\left(\frac{1}{2} n+1\right)$ are naturally dual, being paired by integration. Similarly, the function $\phi \rightarrow(\phi, Y(g) \phi)$ defined on $\mathrm{L}\left(\frac{1}{2} n-1\right)$ by

$$
\phi \rightarrow \int\left[|d f|_{g}^{2}+\frac{1}{4} \frac{n-2}{n-1} R(g) f^{2}\right] d \operatorname{vol}_{g}
$$

extends (by polarizing) to define an operator

$$
\Upsilon(g): \mathbf{L}\left(\frac{1}{2} n-1\right) \rightarrow \mathbb{L}\left(\frac{1}{2} n+1\right) .
$$

2.4. With this notation then we can continue the summary of the results of Bonora, Cotta-Ramusino, and Reina as follows: When $n=4$, the space of $\mathfrak{D}$ equivariant elements of

$$
H^{1}\left(\mathfrak{w}, \Omega^{0}\left(\mathfrak{M} \times \mathbb{L}\left(\frac{1}{2} n-1\right)\right)\right)
$$

is four-dimensional, having in addition to $C_{\mathrm{I}} \otimes 1$ and $C_{\mathrm{II}} \otimes 1$ the basic elements

$$
1 \otimes C_{\mathrm{III}}=|\phi|^{2 n /(n-2)}
$$

as well as

$$
C_{\mathrm{IV}}=\phi Y(g) \phi
$$

Thus in the physics literature one often reads that the real scalar boson, conformally coupled to gravitation, has anomalies. In particular this means that these groups contain one more cohomology class then the Künneth formula would predict.

On the other hand, it is clear that

$$
T c_{1}(\text { ind } Y) \in H^{1}\left(\mathfrak{w}, \Omega^{0} \mathfrak{M}\right)
$$

(not $H^{1}\left(\mathfrak{w}, \Omega^{0}\left(\mathfrak{M} \times \mathbf{L}\left(\frac{1}{2} n-1\right)\right)\right)$ !) and that $C_{\mathrm{IV}}$ does not obstruct the existence of a covariant propagator for $Y(g)$.

This kind of problem has a long history (cf. [13]; see also [6] for some related questions). T. Branson and B. Ørsted display these classes as coefficients of the heat kernel expension for the operator $Y$, in Conformal indices of Riemannian manifolds, Compositio Math. (to appear). 


\section{Construction.}

3.0. We need to be more explicit. In particular the quadratic form defined in $\S 2.3$ defines a Poincaré complex (cf. [22])

$$
0 \rightarrow \mathbb{L}\left(\frac{1}{2} n-1\right) \stackrel{\Upsilon}{\rightarrow} \mathbb{L}\left(\frac{1}{2} n+1\right) \rightarrow 0
$$

of Banach spaces, and the homology $H_{0}=\operatorname{ker} \Upsilon, H_{1}=$ coker $\Upsilon$ inherits the duality $x, y+\operatorname{im} \Upsilon \rightarrow y(x)$, so

$$
\text { ind } \begin{aligned}
\Upsilon & =\operatorname{ker} \Upsilon-\operatorname{coker} \Upsilon \\
& =\operatorname{coker} \Upsilon^{*}-\operatorname{ker} \Upsilon^{*} \quad\left(\text { since } H_{0} \otimes H_{1} \rightarrow R\right. \text { is a perfect pairing) } \\
& =-\left(\operatorname{ker} \Upsilon^{*}-\operatorname{coker} \Upsilon^{*}\right)=-\operatorname{ind} \Upsilon^{*} \\
& =-\operatorname{ind} \Upsilon \quad(\text { since } *=1 \text { on } K O)
\end{aligned}
$$

is an element of order at most two.

Consequently it is very natural to ask if the complex defined by $\Upsilon$ contains a dense subcomplex of the form

$$
0 \rightarrow V \oplus V^{*} \rightarrow V \oplus V^{*} \rightarrow 0
$$

this question is closely connected to the global properties of the Sobolev embedding, and to the existence of a positive-negative frequency decomposition, or polarization, for the quadratic space $\left(\mathbb{L}\left(\frac{1}{2} n-1\right), Y(g)\right)$. We return to this question in $\S 4$.

3.1. In the remainder of this section we write down an integral formula for the symbol $\sigma(\Upsilon) \in K O\left(T^{+} M \times_{\mathfrak{D}_{0}} \mathfrak{C}_{0}\right)$ of the elliptic family $\Upsilon$. Here $T^{+} M$ is the onepoint-compactification of the cotangent bundle $T^{*} M$, and the index-along-the-fiber map

$$
\text { ind: } K O\left(T^{+} M \times_{\mathfrak{D}_{0}} \mathfrak{C}_{0}\right) \rightarrow K O\left(\mathrm{pt} \times \times_{\mathfrak{D}_{0}} \mathfrak{C}_{0}\right)
$$

sends $\sigma(\Upsilon)$ to ind $\Upsilon$.

It will be convenient to think of $T^{*}$ in its real projective compactification $P\left(T^{*} \oplus 1\right)$, as in [3], and to think of a class in $K O\left(T^{+} M\right)$ as the image of a class in $K O(P, P-0), 0$ representing the zero-section of $P$.

3.2. Now sections of the real line bundle $\eta^{\otimes k}$ over the real projectification $P(V)$ of a vector space $V$ can be identified with functions

$$
f: V-0 \rightarrow \mathbb{R}
$$

such that $f(t v)=t^{-k} f(v), t \in \mathbb{R}^{x}$. In particular, a Riemannian inner product on $V=T^{*}$ defines a nonvanishing function $v \rightarrow|g(v)|^{2}$, and consequently a nonvanishing section of $\eta^{\otimes(-2)}$. (It will be useful to index the positive symmetric bilinear forms on $V$ by elements of $O_{n} / \mathrm{Gl}_{n}$, denoted $g \rightarrow|g(v)|^{2}$.) It will also be useful to think of the length-squared as a $\mathrm{Gl}_{n}$-equivariant function

$$
O_{n} \backslash \mathrm{Gl}_{n} \times \eta^{\otimes(-2)} \rightarrow \mathbb{R}(2)
$$

so that the pullback of $\mathbb{R}(d)$ to $P\left(T^{*}\right)$ can be identified with $\eta^{\otimes d}$. Now $\eta^{\otimes k}$, minus its zero-section, is a principal $\mathbb{R}^{\times}$-bundle, with

$$
\theta_{k}(g, v)=k d \log |g(v)|
$$

as natural connection one-form. Note that its curvature is zero. 
3.3. When $n$ is even, e.g. 4 , the complex

$$
0 \rightarrow \eta^{\otimes n / 2+1} \stackrel{\sigma}{\rightarrow} \eta^{\otimes n / 2-1} \rightarrow 0
$$

over $O_{n} \backslash \mathrm{Gl}_{n} \times P(V)$ defined by $\sigma(g, v)=(\operatorname{det} g)^{n^{-1}}|g(v)|^{2}$ is not only $\mathrm{Gl}_{n^{-}}$ equivariant, but $\mathfrak{W}$-equivariant (when $V=T^{*}$ ) as well. The product bundle

$$
\left(\left(O_{n} \backslash \mathrm{Gl}_{n}\right) \times P\left(T^{*} \oplus 1\right)\right) \times \mathrm{Gl}_{n} \mathrm{Fr}
$$

can be understood as the pullback $B \times{ }_{M} P\left(T^{*} \oplus 1\right.$ ) (over the total space = $\left(O_{n} \backslash \mathrm{Gl}_{n}\right) \times \mathrm{Gl}_{n}$ Fr of the bundle of Riemannian metrics) of the bundle of projectifications, and the complex just constructed transplants equivariantly to define a complex over $B \times{ }_{M} P\left(T^{*} \oplus 1\right)$, exact away from the 0 -section $B=B \times_{M} P(0 \oplus 1)$, (cf. [16]). The evaluation map

$$
e: \mathfrak{M} \times M \rightarrow B
$$

pulls this complex back to define a $\mathfrak{D}$-equivariant complex on $P\left(T^{*} \oplus 1\right)$ exact away from zero as above; but in fact the construction is $\mathfrak{W}$-equivariant as well, and descends to define the promised symbol.

3.4. Chern forms for this complex can be constructed using a Quillen superconnection [23] on the $Z_{2}$-graded vector bundle $\eta^{\otimes n / 2 \pm 1}$ over $P\left(T^{*} \oplus 1\right) \times \mathfrak{C}_{0}$ : if $D$ is the graded connection defined by $\theta_{n / 2 \pm 1}$ and $L$ is the odd-degree endomorphism

$$
\left[\begin{array}{cc}
0 & \sigma \\
-\sigma^{-1} & 0
\end{array}\right]
$$

then $D+t L$ is a superconnection on the graded line bundle, and the differential form $\operatorname{Tr}_{s} e^{(D+t L)^{2}}$ represents $\operatorname{ch} \sigma(\Upsilon)$ in $H^{\mathrm{ev}}\left(T^{+} M \times_{\mathfrak{D}_{0}} \mathfrak{C}_{0}\right)$.

3.5. If $M$ is a spin-manifold, the index formula can be written

$$
\operatorname{ch} \text { ind } \Upsilon=\operatorname{Tr}_{s} \int_{T^{*} M} e^{D^{2}+t[D, L]+t^{2} L^{2}} \hat{A}(g),
$$

but integration over the fiber requires care at the 0 -section because of singularites in

$$
[D, L]=\left[\begin{array}{cc}
0 & d|g(v)|^{2} \\
-d|g(v)|^{-2} & 0
\end{array}\right]
$$

and this expression can be rewritten, using Quillen's formulas, as

$$
-\operatorname{Tr}_{s} \int_{0}^{\infty} \int_{S\left(T^{*} M\right)} e^{-t^{2}+t[D, L]} L \hat{A}(g) d t
$$

integrated over the unit sphere bundle $|g(v)|=1$, where $[D, L]$ simplifies to $\left[\begin{array}{ll}0 & 1 \\ 1 & 0\end{array}\right] d|g(v)|^{2}$.

3.6. In general, if $\alpha=\sum \alpha_{k} d v^{k}$ is a 1 -form and $\omega$ is a $q$-form on $T^{*} M$, both homogeneous, then

$$
\int_{S\left(T^{*} M\right)} e^{-t \alpha} \omega \sim t^{n-1} \int_{B\left(T^{*} M\right)}|v|^{-1} \sum_{n \geq k \geq 1}(-1)^{k} v_{k} \prod_{j \neq k} \alpha_{j} \wedge d v^{1} \wedge \cdots \wedge d v^{n} \wedge \omega
$$

in the case of interest here $\alpha$ is the component along the cotangent bundle of $d|g(v)|^{2}=2 \sum v_{k} d v^{k} ;$ consequently

$$
-\sum(-1)^{k} v_{k} \prod_{j \neq k} \alpha_{j}= \begin{cases}\prod v_{k} & \text { if } n \text { is odd } \\ 0 & \text { when } n \text { is even }\end{cases}
$$


3.7. This argument extends without difficulty to more general vector bundle Laplacians. Its general metaphysical explanation is perhaps as follows: Euclidian quantum field theory leads to measures defined on function spaces, Gaussian measures being the simplest sort. In particular there is such a measure on the space of distribution sections of the bundle of $\left(\frac{1}{2} n-1\right)$-densities, characterized by the Bochner-Minlos transform

$$
e\left(-\pi\left(\phi, Y(g)^{-1} \phi\right)\right)=\int e(2 \pi i \phi \cdot \psi) d \operatorname{Gauss} \Upsilon(\psi)
$$

i.e. with covariance $Y(g)^{-1}$ as a quadratic form on the smooth $\frac{1}{2} n+1$ densities $\phi$. The correspondence between Gauss measures and quadratic forms is functorial (cf. Generalized functions, vol. IV). In particular there are no meaningless factors of $\operatorname{det} Y(g)$ : they are the consequence of an unnatural projection into some Hilbert space. Physicists are in practice interested in regularization procedures for such measures, which motivates the question of gauge-covariant propagators for $Y(g)$.

\section{Some more subtle invariants.}

4.0. Recall that $L_{r}^{p}$ is the completion of the smooth real-valued functions in the norm $f \rightarrow\left\|\left(-\Delta_{g}+1\right)^{r / 2}\right\|_{p}$.

When $r-n p^{-1} \geq s-n q^{-1}$, the Sobolev theorems assert the existence of a dense embedding $L_{r}^{p} \rightarrow L_{s}^{q}$. Specializing to the case $p=2, r=1, s=0$ we obtain the sequence

and the dual

$$
0 \rightarrow L_{1}^{2} \rightarrow L_{0}^{2 n /(n-2)} \rightarrow 0
$$

$$
0 \rightarrow L_{0}^{2 n /(n+2)} \rightarrow L_{-1}^{2} \rightarrow 0,
$$

weakly exact in that the image of each morphism is dense in the kernel of the succeeding one. Splicing yields the short weakly exact sequence

$$
0 \rightarrow L_{0}^{2 n /(n+2)} \rightarrow L_{-1}^{2} \oplus L_{+1}^{2} \rightarrow L_{0}^{2 n /(n-2)} \rightarrow 0 .
$$

4.1. The isomorphism

$$
(x, y) \rightarrow\left(-\Delta_{g}+1\right)^{-1 / 2} x+i\left(-\Delta_{g}+1\right)^{+1 / 2} y: L_{-1}^{2} \oplus L_{+1}^{2} \rightarrow L_{0}^{2} \otimes \mathbb{C}
$$

represents multiplication by $i$ on the complex Hilbert space on the right, as the endomorphism

$$
I(g)=\left[\begin{array}{cc}
0 & -\Delta_{g}+1 \\
-\left(-\Delta_{g}+1\right)^{-1} & 0
\end{array}\right]
$$

of the real vectorspace $L_{-1}^{2} \oplus L_{+1}^{2}$.

For future reference (cf. $[2, \S 1])$, note that besides

(i) $I(q)^{2}=-1$

we have

(ii) $J I(q)=-I(q) J, J=\left[\begin{array}{cc}i & 0 \\ 0 & -i\end{array}\right]$.

4.2. In the notation of $\S 2$ the weakly exact sequence just constructed can be written in the form

$$
0 \rightarrow \mathrm{L}\left(\frac{1}{2} n+1\right) \rightarrow \mathrm{L}\left(\frac{1}{2} n\right) \otimes \mathbb{C} \rightarrow \mathrm{L}\left(\frac{1}{2} n-1\right) \rightarrow 0
$$

in which the spaces do not vary with the Riemannian metric. The middle Hilbert space is a completion of the subspace $C^{\infty} \mathbb{L}\left(\frac{1}{2} n+1\right) \oplus C^{\infty} \mathrm{L}\left(\frac{1}{2} n-1\right)$ of smooth 
$\left(\frac{1}{2} n \pm 1\right)$-densities $z=x+i y$ in $\mathbb{L}\left(\frac{1}{2} n+1\right) \oplus \mathbb{L}\left(\frac{1}{2} n-1\right)$, according to the norm defined by the Hermitian inner product

$$
H_{g}\left(z_{1}, z_{0}\right)=\left[\left(x_{1}, x_{0}\right)_{-1}+\left(y_{1}, y_{0}\right)_{+1}\right]+i\left[\left(x_{1}, y_{0}\right)-\left(x_{0}, y_{1}\right)\right]
$$

where $(-,-)_{s}=\left(-,\left(-\Delta_{g}+1\right)^{s}-\right),(-,-)=(-,-)_{0}$.

4.3. The imaginary part of $H_{g}$ is the standard symplectic form on $\mathbb{L}\left(\frac{1}{2} n+1\right) \oplus$ $\mathbb{L}\left(\frac{1}{2} n-1\right)$, which is defind without reference to $g$. We obtain from a family of metrics a family of Hermitian structures with fixed underlying symplectic Hilbert space, and thereby a map

$$
(\text { Metrics }) \longrightarrow(U / \mathrm{Sp})\left(\mathbb{L}\left(\frac{1}{2} n\right) \otimes \mathbb{C}\right) .
$$

Indeed, if $g_{0}$ and $g_{1}$ are two possible metrics, then the operator

$$
\left[\begin{array}{cc}
\left(-\Delta_{g_{1}}+1\right)^{1 / 2}\left(-\Delta_{g_{0}}+1\right)^{-1 / 2} & 0 \\
0 & \left(-\Delta_{g_{1}}+1\right)^{-1 / 2}\left(-\Delta_{g_{0}}+1\right)^{+1 / 2}
\end{array}\right]
$$

which transports $H_{g_{1}}$ to $H_{g_{0}}$ is a pseudodifferential operator of order zero with leading symbol 1 ; it is thus of the form $1+$ Compact, and we obtain a map

$$
(\text { Metrics }) \longrightarrow(U / \mathrm{Sp})_{c}\left(\mathbb{L}\left(\frac{1}{2} n\right) \otimes \mathbb{C}\right)
$$

to a subspace of the larger Lagrange Grassmannian; the space $(U / \mathrm{Sp})_{c}$ is homotopy equivalent to $\Omega^{3} B O$ and thus represents the functor $K O^{5}$ (cf. $[\mathbf{2}, \mathbf{4}, \mathbf{1 8}$, Lemma 24.6]).

4.4. In fact this construction defines a class in $K O^{5}\left(\mathfrak{M} / \mathfrak{D}_{0}\right)$, and by replacing $-\Delta_{g}+1$ with the absolute value

$$
\left|-\Delta_{g}+\frac{1}{4} \frac{n-2}{n-1} R(g)\right|
$$

(and attending to its possible kernel and cokernel) we can construct a sharper invariant in $K O^{5}\left(\mathfrak{C}_{0} / \mathfrak{D}_{0}\right)$. Of course

$$
K O^{n}(X) \otimes \mathbb{Q}=\bigoplus_{i \equiv n(8)} H^{i}(X, \mathbb{Q}),
$$

but index theory does not seem to shed much light on this sort of invariant.

\section{REFERENCES}

1. O. Alvarez, I. M. Singer and B. Zumino, Gravitational anomalies and the families' index theorem, Comm. Math. Phys. 96 (1984), 409-417.

2. V. I. Arnol'd, Characteristic classes entering in quantization conditions, Functional Anal. Appl. 1 (1967), 1-13.

3. M. F. Atiyah, $K$ theory, Benjamin, New York, 1968.

4. M. F. Atiyah and I. M. Singer, Index theory for skew-adjoint Fredholm operators, Inst. Hautes Études Sci. Publ. Math. 37 (1969), 5-26.

5. _ Dirac operators coupled to vector potentials, Proc. Nat. Acad. Sci. U.S.A. 81 (1984), 2597-2601.

6. D. Bao and V. P. Nair, A note on the covariant anomaly as an equivariant momentum mapping, Comm. Math. Phys. 101 (1985), 437-448.

7. W. A. Bardeen and B. Zumino, Consistent and covariant anomalies in gauge and gravitational theories, Nuclear Phys. B 144 (1984), 421-453.

8. L. Bonora and P. Cotta-Ramusino, Some remarks on BRS transformations, anomalies, and the cohomology of gauge groups, Comm. Math. Phys. 87 (1983), 589-603. 
9. L. Bonora, P. Cotta-Ramusino and C. Reina, Conformal anomaly and cohomology, Phys. Lett. B 126 (1986), 305-308.

10. T. Branson, Differential operators canonically associated to a conformal structure, Math. Scand. 57 (1985), 293-345.

11. S. S. Chern and J. Simons, Characteristic forms and geometric invariants, Ann. of Math. (2) 99 (1974), 48-69.

12. D. Ebin, The space of Riemannian metrics, Proc. Sympos. Pure Math., vol. 15, Amer. Math. Soc., Providence, R.I., 1970, pp. 11-41.

13. F. Englert, R. Gastmans and C. Truffin, Conformal invariance in quantum gravity, Nuclear Phys. B 117 (1976), 407-432.

14. G. Falqui and C. Reina, BRS cohomology and topological anomalies, Comm. Math. Phys. 102 (1985), 503-575.

15. A. E. Fischer and J. F. Marsden, The manifold of conformally equivalent Riemannian metrics, Canad. J. Math. 29 (1977), 193-209.

16. K. Fujikawa, Comment on chiral and conformal anomalies, Phys. Rev. Lett. 44 (1980), 17331736.

17. D. J. Gross and R. Jackiw, Effect of anomalies on quasirenormalizable theories, Phys. Rev. D 6 (1972), 477-493.

18. J. Milnor, Morse theory, Ann. of Math. Studies, no. 51, Princeton Univ. Press, Princeton, N.J., 1963.

19. J. Morava, Conformal invariants of Minkowski space, Proc. Amer. Math. Soc. 95 (1985), 565570.

20. R. S. Palais et al., Seminar on the Atiyah-Singer index theorem, Ann. of Math. Studies, no. 57, Princeton Univ. Press, Princeton, N.J., 1965.

21. S. M. Paneitz, Unitarization of symplectics and stability for causal differential equations in Hilbert space, J. Funct. Anal. 41 (1981), 315-326.

22. A. Ranicki, Exact sequences in the algebraic theory of surgery, Math. Notes, no. 26, Princeton Univ. Press, Princeton, N.J., 1981.

23. D. Quillen, Superconnections and the Chern character, Topology 24 (1985), 89-95.

24. E. Witten, Global gravitational anomalies, Comm. Math. Phys. 100 (1985), 197-229.

Department of MAThematics, Johns Hopkins UNiversity, Baltimore, MARYLAND 21218 (Current address of Jack Morava)

Current address (Hirotaka Tamanoi): School of Mathematics, Institute for Advanced Study, Princeton, New Jersey 08544 